\title{
Automated Tablet Quality Assurance and Identification for Hospital Pharmacies
}

\author{
Zenon Chaczko and Anup Kale
}

\begin{abstract}
The tablet quality checking and identification in hospital pharmacies is done manually and does not use any automated solution. Manual sorting and handling makes this activity laborious and error-prone. This paper describes a low cost solution that is characterised by a small size of the infrastructure involved. Discussed are design and implementation details of Tablet Inspection System based on Machine Vision. The described process uses a dedicated sequence of operation to perform dispensing, scanning and sorting using mini factory setup. Machine Vision System uses a novel Genetic Evolution algorithm. The algorithm provides robust and scalable output. Due to its versatile nature and easy shape recognition ability the approach can be easily adapted to a large variety of medical tablets. The proposed solution attempts to follow the concept of single objective with multiple optima in GA that is designed to scan multiple number of tablets in one cycle of operation.
\end{abstract}

Keywords-hospital pharmacy, content error, genetic algorithm.

\section{INTRODUCTION}

$\mathbf{R}$ ECENT advancement in an industrial automation and robotic equipment has enhanced implementation of the process automation in many other domains as well. Hospitals in developed world as well can absorb lot of this automation and enhance efficiency in certain repetitive, laborious and error-prone activities. In this paper we will explore automation for hospitals pharmacies. Main encouragement behind this work is number of errors introduced in the tablet dispensing and quality check process due to manual nature of activity.

Based on research conducted by [1] shows growing concern about number of patients harmed by wrong medication in hospitals. This research provides facts and figures indicating level medication dispensing errors in the USA. Various types of errors in medication including content errors, labelling errors and documentation errors are discussed. In another similar study conducted by [2] throws light on errors introduced during drug distribution process in hospitals from countries UK and Germany. In this work, the focus is on various errors which include omission, wrong drug, wrong dose, extra dose, unauthorised drug dose, wrong dosage form and expired drug are discussed thoroughly.

Based on both these studies prove that medication errors occur in medication process and effects can be fatal depending on error level. Thus based on above discussion it can be concluded that automation can add big value to medication process and can certainly reduce number of errors introduced

Z. Chaczko and A. Kale are with the University of Technology, Sydney, Broadway NSW 2007, Australia (e-mail: Zenon.Chaczko@uts.edu.au; Anup.V.Kale@student.uts.edu.au). in the process. Especially quality check of tablet from drug content point of view needs sophisticated machine vision system to detect errors.

First section describes introductory part with motivations behind work. Section 2 discusses background of problem space by throwing light on sources of medication, tablet defects and errors. In this section we cover all possible defects which can be visible and are detected by machine vision. Section 3 of paper provides details on proposed solution with architectural and algorithmic description of system. Section 4 provides details of experiment called Smart Mini Factory for Quality check of tablets. In this section all results found during experiment are discussed with actual diagrams and graphical analysis. Section 5 of paper provides the future direction of research of Hospital Automation and Evolutionary Machine Vision systems. And last section concludes with conclusions of this paper.

\section{BACKGROUND STUDY}

Hospital medication errors may occur in every step of process. Common steps in Medication include Prescribing, Transcribing, Dispensing, Administering, Monitoring and System Management Control [3]. Prescription error includes Prescription of Medication history at the time of admission, assessment errors and therapeutic response [3], [4]. Dispensing errors constitute content level, labeling and documentation errors [1], [5]. Since most other errors are out of scope of this paper they are not discussed. During this research maximum focus was given on Tablet quality errors.

Out of content errors in dispensing it was found that drug dosage and drug selection as most important factors. Also it was observed that these errors are purely human errors [6]. Automated solution can help to avoid certain problems associated with this area of concern. Especially tablet related problems can be handled by robotic solution, since tablet handling and inspection is relatively feasible with modern robotic equipment.

\section{A. Tablet Defects in Medication}

Wrongly labeled or ambiguous labeling may lead to mistakes and create the damage [5]. It may lead to misinterpretation of drug dosage, or even type of drug by hospital staff. Thus wrongly selected drug supplied to a patient may lead to fatal health problem due to wrong drug dosage and absence of required drug. For these reasons tablet identification is critical. 


\section{1) Tablet Quality}

There is always possibility of physical damage to tablet due to transportation and handling or even rarely manufacturing defects. Categorization of defects [7] can be performed according to the following criteria:

- Tablet Geometry: Dimensions and shape of tablet can be the defect. Especially if dimensions are wrong then net drug content will be also wrong.

- Breakages: In case breakages it not just reduces the content of drug but also creates doubt about reliability of tablet in patient's mind.

- Coating defects: Coating may have one or more of the following purposes [8]; strengthening, test improvement, handling and packaging convenience, and protection from moisture or sometimes even to suppressing strong smells [9]. Any damage to tablet coating will lead to damage to tablet quality and adding contamination to it.

- Aspects: Aspect provides most of the times identification or manufacturer's signature to a particular product. Any damage to aspect leads to tablet identification errors.

- Print: Print as well is important feature from identification point of view. Any print related problem may misguide in selection of drug.

- Embossing: Embossing of tablet provides tablet an identity and useful to distinguish it from other similar tablets [9]. Thus embossing errors also may lead to wrong identification of tablets.

- Expired Medicine: Usage of expired medicine may lead to conical effects of drug and even may lead to serious harm to a patient [10]. So it is important to keep track of expiry date of every drug in hospital.

\section{2) Packaging Errors}

Some of packaging defects identified from this research perspective [6], [11], [12] includes:

- Similar Packages to two distinctly different drugs;

- Similar packaging to two similar named drugs;

- Counting and mixing before packaging;

- Damaged packaging leading to contamination of Tablets.

Referring to information obtained so far this section leads to conclusion that, during packaging process of drugs in hospital pharmacies following major defects occur and they need to be addressed;

- Identification of drug;

- Contamination; and

- Content level of a drug (i.e. this defect can be caused by a broken tablet).

During this paper we propose a Machine Vision based system to address these problems related with Tablets.

\section{3) Tablet Sorting and Distribution Environment}

Since quality of drug also depends on environment of storage and distribution, it is essential to monitor environmental parameters [10]. It is also mandatory legal requirement to maintain pharmacy environment for:

- Damaged packaging leading to contamination of Tablets.

- Clean Room Environment;
- Controlled Storage Environment;

- Maintained environment pressure.

Thus it is essential to monitor environmental parameters to fulfill above requirements. Environmental parameters to be measured include:

- Air content for its quality;

- Environment temperature;

- Pressure level of environment.

Thus this information describes required environment for tablet quality check process.

\section{B. Available Solutions}

There are many full fledge solutions are available to inspect tablet types and quality in pharmaceutical industry. These solutions are made for large scale industries and are very expensive from cost and space point of view. There is lot of algorithmic work done on tablet quality checking [13], [14]. These algorithms rely on classical approach (deterministic and probabilistic) and need stringent requirements of setup.

\section{Methodology}

\section{A. Tablet Description from Machine Vision Perspective}

Tablet as a single unit from machine vision perspective needs to be assessed for size, shape, colour and special marks and aspects. In proposed work we are going to check colour and shape features strictly as we are more concerned about content of drug and its contamination. Special marks, prints and aspects are usually already assessed during manufacturing and small variation will not change drug content drastically. So in this work very little focus is provided on checking these features.

\section{B. Overall Process with Pick and Place Strategy}

Quality checking of tablets in hospital pharmacies assumes few considerations which include maintained environment suitable for Drug storage and handling and tablets without sachet. Thus tablets to be checked can be checked either in batches of same tablet or in mixed assorted group. Mixed group can lead to mix up of tablets at partial or unit level due to powder of broken tablet or mistake committed by Vision System. So here quality check is performed at group of single type tablet. Overall system as a process needs dispensing, conveying (moving by conveyor belt), quality checking and sorting to accepted quality location or rejected location. The system architecture that encapsulates the problem of process control is shown in Figure 1.

\section{Quality Check Process and Algorithm}

Machine vision for tablet quality was developed using single web camera. Two dimensional pattern of each tablet was compared with sample template of same type using robust algorithmic technique based on evolutionary computational principles. 


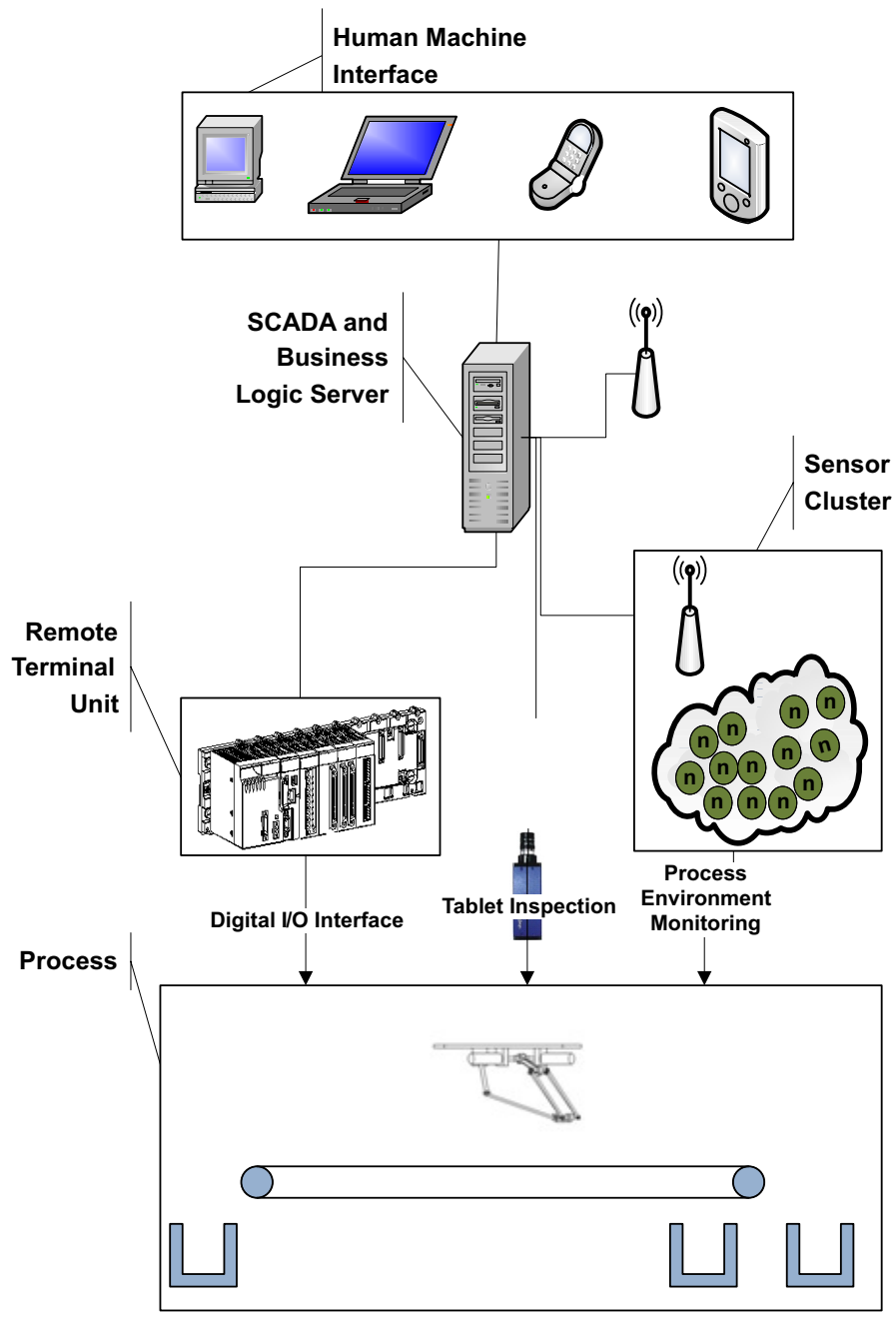

Fig. 1. Process control system architecture.

\section{a) Genetic Algorithms}

Genetic algorithm or GA works on principle of biological evolution [15]. In GA, the principle of "fittest survives" is applied and random population of possible solutions is generated. Every element of population is checked for fitness level. Process of finding solution is called as optimization and performed by checking value of every element of population against desired objective function or fitness level. Effort is also put by several researchers to apply GA for machine vision and image processing applications [16], [17]. In case of machine vision applications GA provides advantage over classical by reducing computational complexity. GA based Machine vision work [14] is importantly growing and will be very useful when large amount of information is handled in short amount of time.

\section{b) Overall Working of Algorithm}

Process starts with initialization of population of possible optimizations. First iteration of loop starts with checking fitness of entire population and counted as first generation. After population check the population with accepted fitness level is omitted from further manipulation. At this point the optimization level is checked and if optimization level is

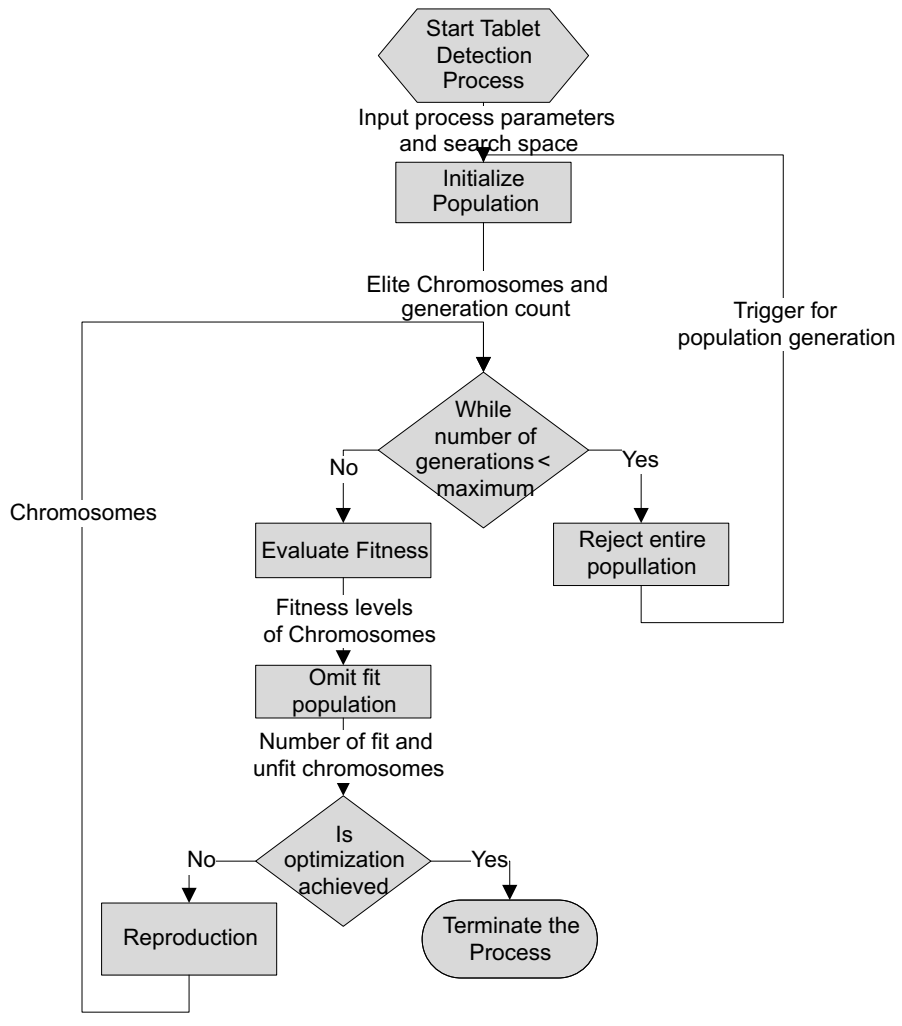

Fig. 2. Flow diagram of the proposed algorithm.

achieved the further processing is terminated. If optimization level not achieved then crossover and mutation is performed to add variation to population. After crossover and mutation population is send again back to fitness check and generation count is incremented. Once number of counts of fitness check reach maximum then entire population is assumed as reject and many tablets are rejected considering below the required quality.

\section{1) Population Initialization}

In this process of algorithm random population of centre points of tablet geometry are generated proportionally to number of tablets dispensed. For reducing computational efforts all centre points below threshold level are rejected. Once elite generation of points is received then population of chromosomes is created by defining Region of interest (ROI) equal to size of template of tablets to be inspected for their physical defects.

All points in square areas represent points above threshold level and square as region of interest. Whereas points in circles are points below threshold level and are not used for further manipulation. If $\mathrm{A}$ is set of centre points generated randomly then, set of Elites E is;

$$
E=\{(x, y), \text { for all } f(x, y)>\text { threshold }\}
$$

\section{2) Individual Encoding}

In our approach two parameters are used to build the chromosome or square shaped Region of Interest (ROI) to find solution. These two parameters are co-ordinate pair $(x, y)$ of centre of ROI. 


\section{3) Fitness Evaluation}

It is achieved by comparing surface area and pixel to pixel intensity difference of low resolution model of Template and chromosomes. Reason behind selecting this methodology is that it provides simple and robust technique to find broken tablets and colour defects.

First step comparing surface area of tablet chromosome with Template:

$$
A=\sum_{(x, y) \in R} f(x, y)
$$

"A" in (2) is formula for calculating area and whereas (3) provides fitness function to check Histogram Intersection [18]. Minimum the value of this function will provide maximum fitness level. Here distance between template and chromosome histograms is measured for assessing similarity between template and chromosome. This approach can measure difference between colour density patterns of two different regions.

$$
D_{H I}(t, c)=\sum_{i=0}^{M-1}\left|h_{t}(i)-h_{c}(i)\right|
$$

Omission of fit chromosomes: In this process chromosomes above desired fitness level are considered as partially optimized and are not considered for further manipulation. Thus with every cycle of 'fitness checking' tablets with desired quality are omitted from further processing. If at the start of genetic search, number of tablets on conveyor belt $=m$. After one fitness checking is performed tablets with above fitness found $=n$; then after one cycle of fitness checking tablets remaining on conveyor belt $=(m-n)$.

\section{4) Reproduction}

Crossover: Crossover process produces off-springs by selecting from set of chromosomes sorted as per fitness order. In crossover last two bits of $\mathrm{x}$ and $\mathrm{y}$ co-ordinates of elite point population are swapped with each other to create drift in position of ROI. In this case, we are performing two point crossover and swapping every last 3 bits of $\mathrm{x}$ and $\mathrm{y}$ co-ordinate in population.

\section{5) Mutation}

If after certain number of cycles population converges to local maxima and not able to produce optimization then mutation is performed. In this case mutation creates random bit modification to change population completely. In this experiment we swapped bit 7 of $\mathrm{x}$ with bit 1 of $\mathrm{y}$ and vice versa was sapped.

Rejection of Population: If process fails to find optimization after certain number of crossover and mutation cycles then set of chromosomes which represent set of tablets on conveyor is assumed as unfit population and is rejected. After rejecting population the new population generation process is triggered.

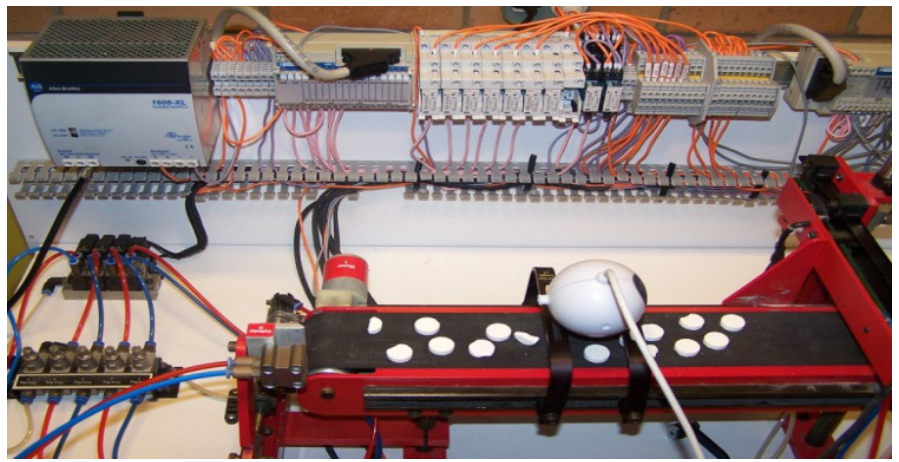

Fig. 3. Smart factory setup.

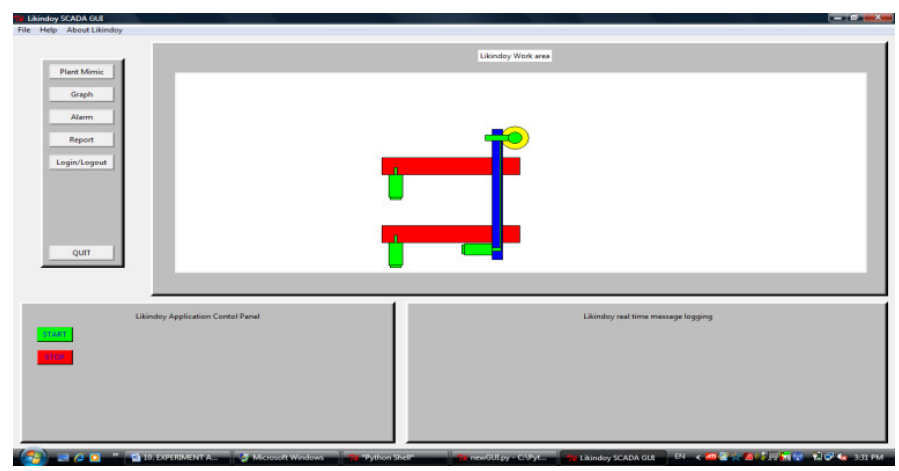

Fig. 4. Plant mimic screen of smart factory.

\section{SMart Factory-CASE Study}

Experiments were carried out for checking versatility, accuracy and scalability of proposed system. Setup for experiment consisted of mini factory setup called Petra (brand name). This mini factory consists of small conveyor belt with pick and place facility. Figure 3 shows photograph of mechanical setup of Petra. Process control system of this setup was developed using Schneider Electric Unity Pro L software and Modicon Programmable Logic Controller of same manufacturer. Figure 4 shows plant mimic screen developed for this experiment.

\section{EXPERIMENTAL RESUlts}

In the experimental set up the following assumptions were made:

- Here attempt is given to low cost and efficient solution to provide good enough tablet quality checking (Not

TABLE I

OPTIMIZATION RESULTS OF TABLET 'A'

\begin{tabular}{cccc}
\hline \hline $\begin{array}{c}\text { Population } \\
\text { size in Pixels }\end{array}$ & $\begin{array}{c}\text { Fitness } \\
\text { level }\end{array}$ & $\begin{array}{c}\text { Number of } \\
\text { tablets } \\
\text { dispensed in } \\
\text { one cycle }\end{array}$ & $\begin{array}{c}\text { Error } \\
\text { percentage }\end{array}$ \\
\hline 5000 & $\begin{array}{c}A_{d}=0.10 \\
I_{d}=0.6\end{array}$ & 1 & $4 \%$ \\
5000 & $\begin{array}{c}A_{d}=0.10 \\
I_{d}=0.6\end{array}$ & 2 & $4 \%$ \\
5000 & $\begin{array}{c}A_{d}=0.10 \\
I_{d}=0.6\end{array}$ & 3 & $3 \%$ \\
5000 & $\begin{array}{c}A_{d}=0.10 \\
I_{d}=0.6\end{array}$ & 4 & $4 \%$ \\
&
\end{tabular}


TABLE II

OptimizATION RESUlts OF TABLET 'B'

\begin{tabular}{cccc}
\hline \hline $\begin{array}{c}\text { Population } \\
\text { size in } \\
\text { Pixels }\end{array}$ & $\begin{array}{c}\text { Fitness } \\
\text { level }\end{array}$ & $\begin{array}{c}\text { Number of tablets } \\
\text { dispensed checked } \\
\text { in one cycle }\end{array}$ & $\begin{array}{c}\text { Error } \\
\text { percentage }\end{array}$ \\
\hline 5000 & $\begin{array}{c}A_{d}=0.10 \\
I_{d}=0.6\end{array}$ & 7 & $8 \%$ \\
& & \\
\hline \hline
\end{tabular}

matching performance of Pharmaceutical factory level QA).

- During this paper quality of tablet was checked from drug content and contamination point of view only. So any breakages (profile damage), surface damages and contamination on surface of tablets were checked.

- While checking surface damage and contamination rather than checking each and every pixel we are checking number of pixels of tablet image with reduced size equal to actual dimensions of tablets in millimeter vs. resolution. Reason behind this is we are trying to find errors recognizable to human eyes only. Checking each and every pixel leads to very expensive from computational point of view and is unnecessary.

- At the moment only two dimensional profile of tablet was assessed for quality, but in future we will be expanding it to three dimensions for making system more accurate.

- Tablet handling and storage needs specially controlled environment by controlling temperature, pressure air quality. So in this work we are assuming that the location in hospital where this solution will be implemented, that it will already fulfill these environmental requirements.

- In case of tablets other than circular shape they were dispensed on conveyor belt approximately parallel to moving edge of belt for avoiding rotation matrix in calculation part to improve performance.

- Implementation was checked using MATLAB Simulation and ATH 64 QL60 CPU. But in future we would like to port same system on dedicated embedded system.

During this exercise we scanned and checked more than 15 types of tablets and its 80 images for quality check. Results with different population size, fitness levels and number of tablets checked in one genetic optimization cycle.

\section{A. Tabulated Results}

a. Tablet: Round shaped with coloured surface, diameter $=$ $15 \mathrm{~mm}$. Conveyor belt area scanned $=80 \times 120 \mathrm{~mm}$.

b. Tablet: Round shape with white colour Diameter $=12 \mathrm{~mm}$.

Tables 1 and 2 discuss results obtained during the testing of Algorithm. As we can see here very low percentage failure was observed in detection of exact results (2\% to $8 \%$ ). Reason behind these errors was due to conflict created by tablet color. Especially white tablets with small surface damage were not detected by system. And this was due to the fact that two dimensional machine vision system and it fails to detect small variations intensity gradient.

\section{B. Performance of Algorithm}

Based on above results following performance was plotted and it surely indicates that this Algorithm can provide good
TABLE III

VISUALIZATION OF ACCEPTED AND REJECTED TABLETS

\begin{tabular}{|c|c|c|}
\hline Template & $\begin{array}{c}\text { Accepted Tables } \\
\text { After Quality Check }\end{array}$ & $\begin{array}{c}\text { Rejected } \\
\text { Tables }\end{array}$ \\
\hline & & \\
\hline
\end{tabular}

scalability and versatility (Fig. 5). Based on number of experiments performed using different types of tablets with variety in size, shape and colour rigorous testing was performed. As we can observe speed of Algorithm depends on size of optimization results or number of tablets dispensed. But interesting fact is in this case optimization time graph moves towards saturation after certain amount of tablets.

\section{CONCLUSION}

The tablet quality checking process that uses an automated solution provides an answer to problems associated with hospital pharmacies. This approach can be further refined by using the Pareto's optimality approach for planning demand and supply needs. Feature synthesis and analysis of tablets using the Genetic Algorithm based approach provides impressive and detailed results of the process. This scalability, accessibility, accuracy and performance of this solution can be further enhanced by using the latest technologies like

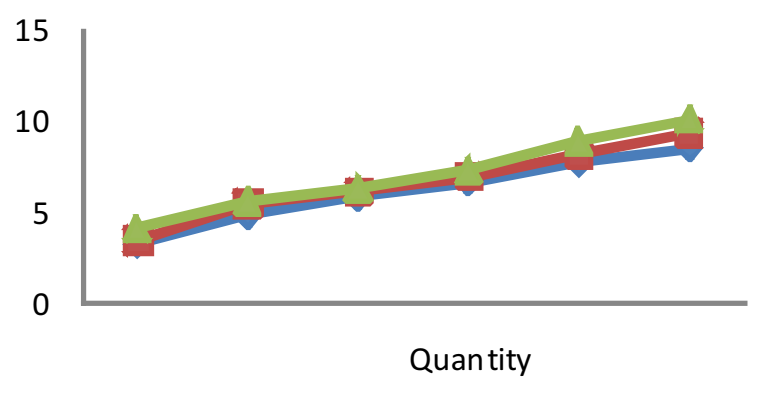

$\longrightarrow$ Tablet 'a' $\longrightarrow$ Tablet 'b'

Fig. 5. Scalability and versatility checking of algorithm. 
high speed equipment, Web Services, stereo vision and multiobjective Genetic Algorithms.

\section{REFERENCES}

[1] A. Besso, B. D. Franklin, and N. Barber, "Frequency and Potential Causes of a Dispensing Error in Hospital Pharmacy," Pharm World Sci, pp. 182-190, 2005.

[2] K. Taxis, B. D. Franklin, and N. Barber, "Hospital Drug Distribution Systems in the UK and Germany - A Study of Medication Errors," Pharm World Sci, vol. 21, no. 1, pp. 25-31, 1999.

[3] A. Berman, "Reducing Medication Errors Through Naming, Labeling, and Packaging," Journal of Medical Systems, vol. 28, no. 1, pp. 10-11, February 2004.

[4] J. R. Pippins, T. K. Gandhi, C. Hamann, C. D. Ndumele, S. A Labonville, E. K. Diedrichsen, M. G. Carty, A. S. Karson, I. Bhan, C. M. Coley, C. L. Liang, A. Turchin, P. C. McCarthy, and J. L. Schnipper, "Classifying and Predicting Errors of Inpatient Medication Reconciliation," Journal of General Internal Medicine, pp. 1414-1415, June 192008

[5] T. Higgins, "Tablet Design: A Critical Aspect of Product Development," Innovations in Pharmaceutical Technologies, vol. 18, pp. 74-76, 2005.

[6] K. Taxis and N. Barber, "Causes of Intravaneous Medication Errors: An Ethnographic Study," Qual Saf Health Care, pp. 33-347, 2003.

[7] M. Zimmerman, "Associated content," July 202005.

[8] P. Aspden, Preventing Medication Errors. Washington DC: The National Academies Press, 2007.
[9] National Coordinating Committee on Therapeutic Goods. Dept. of Health NSW, 1993.

[10] U. Langer and I. Krämer, "GMP in Hospital Pharmacy: Environmental Monitoring of the CIVA Preparation Area," Environmental Health Perspectives, vol. 7, no. 3, pp. 97-107, October 2001.

[11] D. Kopec, M. H. Kabir, D. Reinharth, O. Rothschild, and J. A. Castiglione, "Human Errors in Medical Practice: Systematic Classification and Reduction With Automated Information Systems," Journal of Medical Systems, vol. 27, no. 4, pp. 297-300, August 2003.

[12] H.-J. Guchelaar, M. D. Kalmeijer, and M. E. P. Jansen, "Medication Error Due to Ambiguous Labelling of a Commercial Product," Pharm World Sci, vol. 26, pp. 10-11, 2004.

[13] M. Mozina, D. Tomjevic, F. Pernus, and B. Likar, "Real Time Image Segmentation of Pharmaceutical Tablets," Machine Vision and Applications, August 2009.

[14] E. Deutschl and A. Linnhofer, "Tablet Quality Assurance in Real Time," 14th International Conference on Pattern Recognition, vol. 2, 1998.

[15] J. R. Koza, Genetic Programming. Masschusetts, USA: MIT Press, 1992.

[16] T. Centeno, H. Lopes, M. Felisberto, and L. Arruda, "Object Detection Using Robust Genetic Algorithm, Evo Workshops," LNCS 3449, pp. 284-293, 2005.

[17] R. Cucchiara, "Genetic Algorithms for Clustering in Machine Vision," Machine Vision and Applications, vol. 11, pp. 1-6, 1998.

[18] J. R. Smith and S. F. Chang, "Integrated Spatial and Feature Image Querry," Multimedia Sytems, vol. 7, no. 2, pp. 129-140, 1999. 


\section{VER $\mathbf{E}$ |T $\boldsymbol{T}$ | emerging science publishers}

\section{Serials}

\section{Journal Author}

Versita > Journal Author > FAQ

FAQ

\section{Frequently Asked Questions}

1. What is Versita Open ?

Versita Open is a distribution platform for journals, which Versita publishes in Open Access models. The two Open Access models supported by Versita are author-pays model and publisher-pays model.

All journals at Versita Open offer unrestricted access to readers and libraries. They are peer-reviewed and publish exclusively or predominantly in English. As a rule, authors retain copyright in their works. Versita Open delivers article abstracts to many abstracting databases and delivers full-text to digital repositories and distributors.

\section{What is the difference between the author-pays and publisher-pays models?}

In the publisher-pays model, neither the reader pays for access to the content, nor the author pays for publishing in the journal, thus the publishing costs are borne by the journal owner. In the author-pays model, readers do not pay for access to the content, and publishing costs are borne by the organization to which the author belongs.

If you want to submit a manuscript to one of the journals available at Versita Open, please visit the journal webpage and check if the journal charges publication fees.

\section{Why publish in Versita Open journals?}

Here are the advantages of publishing in a journal available at Versita Open:

Open Access means unrestricted access to published articles. It brings more readers, more citations, and greater awareness of your publication.

Versita Open journals promise fair, fast and comprehensive peer-review.

Most Versita Open journals allow the author to retain copyright. Please visit the journal webpage and check if this applies to the journal of your choice.

Versita applies to all applicable abstracting databases to index each journal, and then supply the abstracts to them, so the abstract of your article will be widely findable.

Versita deposits the abstract of your article at CrossRef, so citations to your article can have an active link, which can be clicked and lead to the abstract of your article.

Versita will send (or make available otherwise) the full-text of your article to numerous repositories (like PubMed Central), distributors and libraries worldwide.

Versita Open is based on MetaPress hosting platform, which lets readers enjoy all the functionalities offered my modern publishing technology solutions. Journals Readers can, for instance, enjoy reference linking. Most references in your article can become active links, which can be clicked to jump to abstracts of cited articles. some journals at Versita Open use Editorial Manager online submission and peer-review system, which is used by more than 4,000 journals, so is known to many authors and reviewers.

\section{Are journals at Versita Open peer-reviewed?}

All journals available at Versita Open are peer-reviewed. Except submissions that are out of scope or of unacceptably low standard, as a rule, all submissions are reviewed by at least two experts in the field. The editors declare fair, fast and comprehensive peer-review.

\section{Do Versita Open journals have Impact Factors ?}

Many of the journals published at Versita Open are newly established journals. So they have not got Impact factors yet. For any journal to have an Impact factor, it must have been tracked by Thomson Scientific for three years. Here are some of Versita Open journals that already have Impact Factors:

Acta Biologica Cracoviensia s. Botanica 0.586

Acta Pharmaceutica 1.312

Acta Physica Slovaca 3.250

Advances in Medical Sciences 0.798

Archives of Acoustics 0.504

Archives of Industrial Hygiene and Toxicology 0.826

Bulletin of the Polish Academy of Sciences Technical Sciences 0.945

Folia Histochemica et Cytobiologica 0.902

Geologica Carpatica 0.909

International Journal of Applied Mathematics and Computer Science 0.794

Journal of Applied Biomedicine 1.689

Journal of Hydrology and Hydromechanics 0.553

Metrology and Measurement Systems 0.587 
Polish Journal of Veterinary Sciences 0.507

Polish Polar Research 0.767

Radiology and Oncology 1.970

\section{What does it cost to publish in Versita Open journal ?}

For the vast majority of Versita Open journals it costs nothing. Because those journals are published in the publisher-pays model, neither the reader pays for access to the content, nor the author pays for publishing in the journal, as the publishing costs are borne by the journal owner. If you want to submit a manuscript to one of the journals available at Versita Open, please visit the journal webpage and check if the journal charges publication fees.

Those Versita Open journals, which are published in author-pays model, apply Article Processing Charges (APC), which are paid by the author's affiliate institution or another funding body. The APC amount depends on the journal and should be checked before submitting a manuscript. Each charging journal has a special waiver policy for authors from low income countries. Waivers are also granted on case-by-case basis.

\section{Why should I have to pay to publish my paper?}

It costs money to produce a peer-reviewed, edited, and formatted article for online publication, and to host it on a server that's accessible around the clock. This cost is small relative to the funding for the research project as a whole (usually around 1\%), but it serves the crucial final step of disseminating a completed body of work to the scientific community. Several major funding sources have explicitly endorsed the open access model for publishing, so it may not be too hard for you to obtain funding for the Article Processing Charge.

\section{Do authors have to assign copyright?}

As a rule, authors retain copyright. However, please visit the journal webpage and check if this applies to the journal of your choice. Most Versita Open journals are published on the basis of Creative Commons license.

Partners

$\underline{\underline{Q}}$
Home
요 3

Members

\section{crossef stm}

ALPSP 